EUROPEAN JOURNAL OF PURE AND APPLIED MATHEMATICS

Vol. 11, No. 4, 2018, 1177-1190

ISSN 1307-5543 - www.ejpam.com

Published by New York Business Global

\title{
Common Fixed Point Theorems in Metric spaces with Applications
}

\author{
Pushpendra Semwal ${ }^{1, *}$, Komal ${ }^{1}$ \\ 1 Department of Mathematics, SoPS, Doon University, Dehradun, India
}

\begin{abstract}
In this paper, we investigate the existence and uniqueness of common fixed point theorems for certain contractive type of mappings. As an application the existence and uniqueness of common solutions for a system of functional equations arising in dynamic programming are discuss by using the our results.
\end{abstract}

2010 Mathematics Subject Classifications: 49L20, 49L99, 54H25, 90C39

Key Words and Phrases: Common fixed point, asymptotically continuous, weakly compatible mapping.

\section{Introduction}

Bellman and Lee [3] first introduced the basic form of the functional equations in dynamic programming is as follows:

$$
f(x)=o p t_{y \in D} H(x, y, f(T(x, y))) \forall x \in S
$$

where opt represent sup. or inf., $x, y$ denote the state and decicion vectors respectively, $T$ stands for the transformation of the process and $f(x)$ represents the optimal return function with the initial state $x$.Afterwards, the existence and uniqueness of fixed point solutions for several classes of contractive mappings and functional equations studied by many investigators such as Bhakta and Mitra [5], Liu [15], Liu and ume [20], Pathak and Fisher [21], Baskaran and Subhramanyam [1] and others.

Ray [22] proved two common fixed point theorems for three self mappings $f, g$ and $h$ in the complete metric space using the following contractive condition:

$$
d(f x, g y) \leq d(h x, h y)-w(d(h x, h y)), \forall x, y \in X
$$

*Corresponding author.

DOI: https://doi.org/10.29020/nybg.ejpam.v11i4.3298

Email addresses: psrsdm@gmail.com (P. Semwal) 
Further Liu[15] established common fixed point theorem and introduced a class of mappings in a complete metric space as follows:

$d(f x, g y) \leq \max \{d(h x, h y), d(h x, f x), d(h y, g y)\}-w(\max \{d(h x, h y), d(h x, f x), d(h y, g y)\})$.

Recall that the notion of orbitally complete metric space and orbitally continuous mapping were introduced by Ciric [9] . These definitions were extended to the case of two or three mappings by Sastry et al.[12]. Some common fixed point results in this situation were obtained in [12]. We give now respective definitions for pairs of mappings given in literature.

\section{Priliminaries}

Definition 1 (6). A self map $f$ on a metric space $(X, d)$ is said to be asymptotically regular at a point $x$ in $X$ if $\lim _{n \rightarrow \infty} d\left(f^{n}(x), f^{n+1}(x)\right)=0$. Where $f^{n}(x)$ denotes the $n^{\text {th }}$ iterate of $f$ at $x$.

Definition 2 (6). Let $f$ and $g$ be two self mappings of $X$ and $\left\{x_{n}\right\}$ a sequence in $X$, then $\left\{x_{n}\right\}$ is said to be asymptotically $g$ - regular with respect to $f$ if $\lim _{n \rightarrow \infty} d\left(f x_{n}, g x_{n}\right)=0$.

Definition 3 (9). Let $\left\{x_{n}\right\}$ is a sequence which is asymptotically g-regular with respect to $f$, then $O\left(f, x_{n}\right)=\left\{f x_{1}, f x_{2}, f x_{3}, \ldots f x_{n}, \ldots\right\}$ is called asymptotic orbit of $f$.

Definition 4 (12). $X$ is said to be $f$-asymptotically complete if every Cauchy sequence of the form $\left\{f x_{n}\right\}$ converges in $X$.

Definition 5 (12). A self map $f$ is said to be asymptotically continuous if it is continuous on closure of $O\left(f, x_{n}\right)$.

Definition 6. Two self maps $g$ and $h$ of $X$ are said to be weakly commuting if $d(g h x, h g x) \leq$ $d(g x, h x) \forall x \in X$

Definition 7 (12). Let $f, g$ and $h$ be three self maps on a metric space $X$.

(i) If for a point $x_{0} \in X$, there exists a sequence $\left\{x_{n}\right\}$ in $X$ such that $f x_{2 n}=h x_{2 n+1}$ and $g x_{2 n+1}=h x_{2 n+2}, n=0,1,2 \ldots$ Then the set $O\left(x_{0}, f, g, h\right)=\left\{T x_{n} \mid n=0,1,2 \ldots\right\}$ is called the orbit of $(f, g, h)$ at $x_{0}$.

(ii) The space $(X, d)$ is said to be $(f, g, h)$ - orbitally complete if every Cauchy sequence in $O\left(x_{0}, f, g, h\right)$ converges in $X$. 
(iii) The map $h$ is said to be $(f, g, h)$ - orbitally continuous at $x_{0}$ if it is continuous on $O\left(x_{0}, f, g, h\right)$.

(iv) The pair $(f, g)$ is said to be asymptotically regular w.r.to $h$ at $x_{0}$ if there exists a sequence $\left\{x_{n}\right\}$ in $X$ such that $f x_{2 n}=h x_{2 n+1}, g x_{2 n+1}=h x_{2 n+2} ; n=0,1,2, \ldots$ and $d\left(h x_{n}, h_{n+1}\right) \rightarrow 0$ as $n \rightarrow \infty$.

Throughout in this paper, we assume that $R^{+}=[0,+\infty), R=(-\infty,+\infty), w$ and $N$ denote the set of all non-negative and positive integers respectively.

$$
W=\left\{w: w: R^{+} \rightarrow R^{+} \text {is continuous mappings with } 0<w(t)<t \forall t>0\right\}
$$

Let $\Phi=\{\phi: \phi:[0, \infty) \rightarrow[0, \infty)\}$ satisfying the following conditions:

(i) $\phi$ is continuous and non-decreasing

(ii) $\phi(t)<t \forall t \in[0, \infty)$

(iii) $\lim _{n \rightarrow \infty} \phi\left(t_{n}\right)=0 \Longleftrightarrow \lim _{n \rightarrow \infty} t_{n}=0$.

Let $\Psi=\{\psi: \psi:[0, \infty) \rightarrow[0, \infty)\}$ satisfying the following conditions:

(i) $\psi$ is non-decreasing

(ii) $\phi(t)<\psi(t) \forall t>0$

(iii) $\psi(a+b) \leq \psi(a)+\psi(b) \forall a, b \in[0, \infty)$

(iv) $\psi(t)<t \forall t \in[0, \infty)$

The aim of this paper is to provide the sufficient conditions for the existence and uniqueness of common fixed point for the following type of contractive mappings metric space $(X, d)$.

$$
\begin{array}{r}
\psi(d(f x, g y)) \leq \max \left\{\phi(d(h x, h y)), \phi\left(d(h x, f x), \phi\left(\frac{1}{2}([d(h x, h y)+d(f x, g y)])\right)\right), \phi(d(h y, g y))\right\} \\
-w\left(\max \left\{\phi(d(h x, h y)), \phi(d(h x, f x)), \phi(d(h y, g y)), \phi\left(\frac{1}{2}([d(h x, h y)+d(f x, g y)])\right)\right\}\right) .
\end{array}
$$

for all $x, y \in X$.Where $\psi$ and $\phi$ are defined above.

As an applications, we discuss the existence and uniqueness of common solutions of the following functional equations arising in dynamic programming.

$$
f(x)=\operatorname{opt}_{y \in D}\{u(x, y)+H(x, y, f(T(x, y)))\} \forall x \in S
$$

and

$$
f_{i}(x)=o_{p} t_{y \in D}\left\{u(x, y)+H_{i}\left(x, y, f_{i}(T(x, y))\right)\right\} \forall x \in S \& i \in\{1,2,3\}
$$




\section{Main Results}

Theorem 1. Let $f, g$ and $h$ be three self maps on a metric space $X$ satisfying:

(i) either $f$ commute with $h$ or $g$ commute with $h$.

(ii) there exists $w \in W$ such that (5) hold for all $x, y \in X$.

(iii) The pair $(f, g)$ is asymptotically regular with respect to $h$ at $x_{0}$.

(iv)The space $X$ is $(f, g, h)$-orbitally complete at $x_{0}$ and $h$ is orbitally continuous at $x_{0}$. Then $f, g$ and $h$ have a unique common fixed point in $X$.

Proof Since $(f, g)$ is asymptotically respect to $h$ at $x_{0}$, there exists a sequence $\left\{x_{n}\right\}$ in $X$ such that $f x_{2 n}=h x_{2 n+1}$ and $g x_{2 n+1}=h x_{2 n+2}, n=0,1,2, \ldots$ and $d\left(h x_{n}, h x_{n+1}\right) \rightarrow$ zero as $n \rightarrow \infty$.

Now we show that $h x_{n}$ is Cauchy.On contrary suppose that $h x_{n}$ is not Cauchy, then there exists an $\epsilon>0$ and positive integers $m_{k}$ and $n_{k}$ with $m_{k}<n_{k}$ such that $d\left(h x_{m_{k}}, h x_{n_{k}}\right) \geq \epsilon$ and $d\left(h x_{m_{k}}, h x_{n_{k}-1}\right) \leq \epsilon$ for all $k=0,1,2, \ldots$. Since $d\left(h x_{m_{k}}, h x_{n_{k}}\right) \leq d\left(h x_{m_{k}}, h x_{n_{k}-1}\right)+$ $d\left(h x_{n_{k}-1}, h x_{n_{k}}\right)$.Then we obtain $d\left(h x_{m_{k}}, h x_{n_{k}}\right) \rightarrow \epsilon$ as $k \rightarrow \infty$.

Now there are four cases: (i) $m_{k}$ is even and $n_{k}$ is odd (ii) $m_{k}$ is even and $n_{k}$ is even (iii) $m_{k}$ is odd and $n_{k}$ is even (iv) $m_{k}$ is odd and $n_{k}$ is odd.

Suppose $m_{k}$ is even and $n_{k}$ is odd, we have

$$
\begin{aligned}
\psi\left(d\left(h x_{m_{k}}, h x_{n_{k}}\right)\right) \leq & \psi\left(d\left(h x_{m_{k}}, h x_{m_{k}+1}\right)\right)+\psi\left(d\left(h x_{m_{k}+1}, h x_{n_{k}+1}\right)\right)+\psi\left(d\left(h x_{n_{k}+1}, h x_{n_{k}}\right)\right) \\
\leq & \psi\left(d\left(h x_{m_{k}}, h x_{m_{k}+1}\right)\right)+\max \left\{\phi\left(d\left(h x_{m_{k}}, h x_{k}\right)\right), \phi\left(d\left(f x_{m_{k}}, h x_{m_{k}}\right)\right),\right. \\
& \left.\phi\left(d\left(g x_{n_{k}}, h x_{n_{k}}\right)\right), \phi\left(\frac{1}{2}\left[d\left(h x_{m_{k}}, h x_{n_{k}}\right)+d\left(f x_{m_{k}}, g x_{n_{k}}\right)\right]\right)\right\} \\
- & w\left(\operatorname { m a x } \left\{\phi\left(d\left(h x_{m_{k}}, h x_{k}\right)\right), \phi\left(d\left(f x_{m_{k}}, h x_{m_{k}}\right)\right), \phi\left(d\left(g x_{n_{k}}, h x_{n_{k}}\right)\right),\right.\right. \\
& \left.\left.\phi\left(\frac{1}{2}\left[d\left(h x_{m_{k}}, h x_{n_{k}}\right)+d\left(f x_{m_{k}}, g x_{n_{k}}\right)\right]\right)\right\}\right)+\psi\left(d\left(h x_{n_{k}+1}, h x_{n_{k}}\right)\right)
\end{aligned}
$$

Letting $k \rightarrow \infty$, we obtain

$$
\psi(\epsilon) \leq \phi(\epsilon)-w(\phi(\epsilon))<\phi(\epsilon)
$$

a contradiction.In the remaining cases we have a similar situation.Hence $\left\{h x_{n}\right\}$ is Cauchy.Since $X$ is $(f, g, h)$ orbitally complete at $x_{0}$, it follows that there exist $z \in X$ s.t. $h x_{n} \rightarrow z$ as $n \rightarrow \infty$. Now, again

$$
\begin{aligned}
\psi\left(d\left(f x_{2 n}, g z\right)\right) \leq & \max \left\{\phi\left(d\left(h x_{2 n}, h z\right)\right), \phi\left(d\left(f x_{2 n}, h x_{2 n}\right)\right), \phi\left(d\left(g_{z}, h_{z}\right)\right),\right. \\
& \left.\phi\left(\frac{1}{2}\left[d\left(h x_{2 n}, h z\right)+d\left(f x_{2 n}, g z\right)\right]\right)\right\}-w\left(\left(\operatorname { m a x } \left\{\phi\left(d\left(h x_{2 n}, h z\right)\right), \phi\left(d\left(f x_{2 n}, h x_{2 n}\right)\right),\right.\right.\right. \\
& \left.\left.\left.\phi(d(g z, h z)), \phi\left(\frac{1}{2}\left[d\left(h x_{2 n}, h z\right)+d\left(f x_{2 n}, g z\right)\right]\right)\right\}\right)\right)
\end{aligned}
$$

and

$\psi\left(d\left(f z, g x_{2 n+1}\right)\right) \leq \max \left\{\phi\left(d\left(h z, h x_{2 n+1}\right)\right), \phi(d(f z, h z)), \phi\left(d\left(g_{x_{2 n+1}}, h_{x_{2 n+1}}\right)\right)\right.$, 


$$
\begin{aligned}
&\left.\phi\left(\frac{1}{2}\left[d\left(h z, h x_{2 n+1}\right)+d\left(f z, g x_{2 n+1}\right)\right]\right)\right\}-w\left(\left(\operatorname { m a x } \left\{\phi\left(d\left(h z, h x_{2 n+1}\right)\right), \phi(d(f z, h z)),\right.\right.\right. \\
&\left.\left.\left.-\quad \phi\left(d\left(g x_{2 n+1}, h x_{2 n+1}\right)\right), \phi\left(\frac{1}{2}\left[d\left(h z, h x_{2 n+1}\right)+d\left(f z, g x_{2 n+1}\right)\right]\right)\right\}\right)\right)
\end{aligned}
$$

Taking $k \rightarrow \infty$, we obtain

$$
\begin{aligned}
\psi(d(z, g z)) & \leq \max \left\{\phi(d(z, h z)), \phi(d(z, z)), \phi(g z, h z), \phi\left(\frac{1}{2}[d(h z, z)+d(z, g z)]\right)\right\} \\
& -w\left(\left(\max \left\{\phi(d(z, h z)), \phi(d(z, z)), \phi(d(g z, h z)), \phi\left(\frac{1}{2}[d(h z, z)+d(z, g z)]\right)\right\}\right)\right)
\end{aligned}
$$

and

$$
\begin{aligned}
\psi(d(f z, z)) & \leq \max \left\{\phi(d(z, h z)), \phi(d(z, z)), \phi(d(g z, h z)), \phi\left(\frac{1}{2}[d(h z, z)+d(f z, z)]\right)\right\} \\
& -w\left(\left(\max \left\{\phi(d(z, h z)), \phi(d(z, z)), \phi(d(g z, h z)), \phi\left(\frac{1}{2}[d(h z, z)+d(f z, z)]\right)\right\}\right)\right)
\end{aligned}
$$

Since $h$ is orbitally continuous at $x_{0}$ and $f h=h f$, we infer that $f h x_{2 n}=h f_{2 n} \rightarrow T z$ as $n \rightarrow \infty$.Similarly $g h x_{2 n+1}=h g x_{2 n+1} \rightarrow h z$ as $n \rightarrow \infty$.

Again,

$$
\begin{aligned}
\psi\left(d\left(f h x_{2 n}, g x_{2 n+1}\right)\right) \leq & \max \left\{\phi\left(d\left(h h x_{2 n}, h x_{2 n+1}\right)\right), \phi\left(d\left(f h x_{2 n}, h h x_{2 n}\right)\right)\right. \\
& \left.\phi\left(d\left(g x_{2 n+1}, h x_{2 n+1}\right)\right), \phi\left(\frac{1}{2}\left[d\left(h h x_{2 n}, h x_{2 n+1}\right)+d\left(f h x_{2 n}, g x_{2 n+1}\right)\right]\right)\right\} \\
- & w\left(\operatorname { m a x } \left\{\phi\left(d\left(h h x_{2 n}, h x_{2 n+1}\right)\right), \phi\left(d\left(f h x_{2 n}, h h x_{2 n}\right)\right), \phi\left(d\left(g x_{2 n+1}, h x_{2 n+1}\right)\right),\right.\right. \\
& \left.\left.\phi\left(\frac{1}{2}\left[d\left(h h x_{2 n}, h x_{2 n+1}\right)+d\left(f h x_{2 n}, g x_{2 n+1}\right)\right]\right)\right\}\right)
\end{aligned}
$$

Taking $k \rightarrow \infty$, we obtain

$$
\begin{aligned}
\psi(d(h z, z)) & \leq \max \left\{\phi(d(h z, z)), \phi(d(h z, h z)), \phi(d(z, z)), \phi\left(\frac{1}{2}[d(h z, z)+d(h z, z)]\right)\right\} \\
& -w\left(\left(\max \left\{\phi(d(h z, z)), \phi(d(h z, h z)), \phi(d(z, z)), \phi\left(\frac{1}{2}[d(h z, z)+d(h z, z)]\right)\right\}\right)\right)
\end{aligned}
$$

implies that

$$
\psi(d(h z, z)) \leq \phi(d(z, h z))-w(\phi(d(z, h z))<\phi(d(z, h z))
$$

a contradiction.Hence $h z=z$.Using (8) and (9) together with $T z=z$, we infer that $f z=g z=h z=z$.Further uniqueness of common fixed point can easily prove.

Taking $\psi(t)=t$ and $\phi(t)=h t$ where $<h<1$, we state the following 
Corollary 1. Let $A, B$ and $T$ be self maps on a metric space $(X, d)$ such that $T$ commutes with both $A$ and $B$ and the pair $(A, B)$ is asymptotically regular w.r.to $T$ at $x_{0} \in X, X$ is orbitally complete and $T$ is orbitally continuous at $x_{0}$ and

$$
\begin{aligned}
d(A x, B y) & \left.\leq \phi\left\{\max \{d(T x, T y), d(A x, T x)), d(B y, T y), \frac{1}{2}[d(T x, T y)+d(A x, B y)]\right)\right\} \\
& \left.\left.-w\left(\left(\max \{d(T x, T y), d(A x, T x)), d(B y, T y), \frac{1}{2}[d(T x, T y)+d(A x, B y)]\right)\right\}\right)\right)
\end{aligned}
$$

for all $x, y \in X$. Then $A, B$ and $T$ have unique common fixed point in $X$.

Theorem 2. Let $(X, d)$ be a metric space and $f, g$ and $h$ be self mappings on $X$ such that $f(X) \cup g(X) \subseteq h(X)$.If there exists a $w \in W$ satisfying (5). Then the pair $(f, h)$ and $(g, h)$ have a coincidence point in $X$, provided that (i) $X$ is h-asymptotically complete, (ii) $h$ is asymptotically continuous and (iii) $h$ is weakly commute with $f$ and $g$. Further $f, g$ and $h$ have a unique common fixed point in $X$.

Proof Let $x_{0} \in X$ be any point in $X$. Since $f(X) \cup g(X) \subseteq h(X)$. We choose sequence $\left\{x_{n}\right\} \in X$ such that $f x_{2 n}=h x_{2 n+1}$ and $g x_{2 n+1}=h x_{2 n+2}$ for all $n \in w$.

By (5), we conclude that

$$
\begin{aligned}
\psi\left(d\left(h x_{2 n+1}, h x_{2 n+2}\right)\right)= & \psi\left(d\left(f x_{2 n}, g x_{2 n+1}\right)\right) \\
\leq & \max \left\{\phi\left(d\left(h_{2 n}, h x_{2 n+1}\right)\right), \phi\left(d\left(h x_{2 n}, f x_{2 n}\right)\right), \phi\left(d\left(h x_{2 n+1}, g x_{2 n+1}\right)\right)\right. \\
& \left.\phi\left(\frac{1}{2}\left(\left[d\left(h x_{2 n}, h x_{2 n+1}\right)+d\left(f x_{2 n}, g x_{2 n+1}\right)\right]\right)\right)\right\}-w\left(\operatorname { m a x } \left\{\phi\left(d\left(h_{2 n}, h x_{2 n+1}\right)\right)\right.\right. \\
& \left.\phi\left(d\left(h x_{2 n}, f x_{2 n}\right)\right), \phi\left(d\left(h x_{2 n+1}, g x_{2 n+1}\right)\right), \phi\left(\frac{1}{2}\left(\left[d\left(h x_{2 n}, h x_{2 n+1}\right)+d\left(f x_{2 n}, g x_{2 n+1}\right)\right]\right)\right)\right\}
\end{aligned}
$$

This yields

$$
\begin{aligned}
\psi\left(d_{2 n+1}\right) \leq & \max \left\{\phi\left(d_{2 n}\right), \phi\left(d_{2 n}\right), \phi\left(d_{2 n+1}\right), \phi\left(\frac{1}{2}\left(d_{2 n}+d_{2 n+1}\right)\right)\right\} \\
& -w\left(\max \left\{\phi\left(d_{2 n}\right), \phi\left(d_{2 n}\right), \phi\left(d_{2 n+1}\right), \phi\left(\frac{1}{2}\left(d_{2 n}+d_{2 n+1}\right)\right)\right\}\right) .
\end{aligned}
$$

Suppose $d_{2 n+1}>d_{2 n}$, then $\phi\left(d_{2 n+1}\right)>\phi\left(d_{2 n}\right)$.Using (5), we have

$$
\psi\left(d_{2 n+1}\right) \leq \phi\left(d_{2 n+1}\right)-w\left(\phi\left(d_{2 n+1}\right)\right)<\phi\left(d_{2 n+1}\right)
$$

a contradiction. Consequently, we have $d_{2 n+1} \leq d_{2 n}$, from (5) we have

$$
\psi\left(d_{2 n+1}\right) \leq \phi\left(d_{2 n}\right)-w\left(\phi\left(d_{2 n}\right)\right)<\phi\left(d_{2 n}\right)
$$

for any $n \in w$. Similarly, we have $\psi\left(d_{2 n}\right) \leq \phi\left(d_{2 n-1}\right)-w\left(\phi\left(d_{2 n-1}\right)\right)<\phi\left(d_{2 n-1}\right)$ for all $n \in N$.It follows that

$$
\psi\left(d_{n}\right) \leq \phi\left(d_{n-1}\right)-w\left(\phi\left(d_{n-1}\right)\right) \forall n \in N
$$


From (10), we have

$$
\sum_{i=0}^{n} w\left(\phi\left(d_{i}\right)\right) \leq \phi\left(d_{0}\right)-\psi\left(d_{n}\right)<\phi\left(d_{0}\right) \forall n \in N
$$

Thus the sequence $\left\{d_{n}\right\}$ is decreasing sequence whereas the series $\sum_{n=0}^{\infty} w\left(\phi\left(d_{n}\right)\right)$ and $\left\{\phi\left(d_{n}\right)\right\}$ are convergent.It is clear that $\lim _{n \rightarrow \infty} w\left(\phi\left(d_{n}\right)\right)=0$. Since sequence $\left\{d_{n}\right\}$ is decreasing so there exists $p \in R^{+}$such that $\lim _{n \rightarrow \infty} d_{n}=p$. By continuity of $\phi$ and $w$ we have $\lim _{n \rightarrow \infty} w\left(\phi\left(d_{n}\right)\right)=w(\phi(p))=0$. Thus $p=0$. Therefore $\lim _{n \rightarrow \infty} d\left(h x_{n}, h x_{n+1}\right)=0$ implies that $\lim _{n \rightarrow \infty} d\left(h x_{2 n}, h x_{2 n+1}\right)=0$ means that $\lim _{n \rightarrow \infty} d\left(h x_{2 n}, f x_{2 n}\right)=0$ and $\lim _{n \rightarrow \infty} d\left(h x_{2 n+1}, g x_{2 n+1}\right)=0$. i.e. the sequence $\left\{x_{n}\right\}$ is asymptotically $h$-regular with respect to $f$ and $g$.

Next we show that $\left\{h x_{n}\right\}$ is Cauchy sequence in $X$.We need only to show that $\left\{h x_{2 n}\right\}$ is Cauchy sequence. On contrary suppose $\left\{h x_{n}\right\}$ is not Cauchy.Then there exists some $\epsilon>0$ such that for any even integers $2 m(k)$ and $2 n(k)$ with $2 m(k)>2 n(k)>2 k$ and $d\left(h x_{2 m(k)}, h_{2 n(k)}\right)>\epsilon$. Further, let $2 m(k)$ denote the least even positive integer exceeding $2 n(k)$ which satisfies that $2 m(k)>2 n(k)>2 k$

$$
d\left(h x_{2 m(k)-2}, h x_{2 n(k)}\right) \leq \epsilon \text { and } d\left(h x_{2 m_{k}}, h x_{2 n(k)}\right)>\epsilon .
$$

Note that for any $k \in N$

$$
\begin{gathered}
d\left(h x_{2 m(k)}, h x_{2 n(k)}\right) \leq d_{2 m(k)-1}+d_{2 m(k)-2}+d\left(h x_{2 m(k)-2}, h x_{2 n(k)}\right) . \\
\left|d\left(h x_{2 m(k)}, h x_{2 n(k)+1}\right)-d\left(h x_{2 m(k)}, h x_{2 n(k)}\right)\right| \leq d_{2 n(k)} . \\
\left|d\left(h x_{2 m(k)+1}, h x_{2 n(k)+1}\right)-d\left(h x_{2 m(k)}, h x_{2 n(k)+1}\right)\right| \leq d_{2 m(k)} . \\
\left|d\left(h x_{2 m(k)+1}, h x_{2 n(k)+2}\right)-d\left(h x_{2 m(k)+1}, h x_{2 n(k)+1}\right)\right| \leq d_{2 n(k)+1} .
\end{gathered}
$$

From above inequalities, we infer that

$$
\begin{aligned}
\epsilon & =\lim _{n \rightarrow \infty} d\left(h x_{2 m(k)}, h x_{2 n(k)}\right) \\
& =\lim _{n \rightarrow \infty} d\left(h x_{2 m(k)}, h x_{2 n(k)+1}\right) \\
& =\lim _{n \rightarrow \infty} d\left(h x_{2 m(k)+1}, h x_{2 n(k)+1}\right) \\
& =\lim _{n \rightarrow \infty} d\left(h x_{2 m(k)+1}, h x_{2 n(k)+2}\right) .
\end{aligned}
$$


Again from (5), we have

$$
\begin{aligned}
\psi\left(d\left(f x_{2 m(k)}, g x_{2 n(k)+1}\right)\right) \leq & \max \left\{\phi\left(d\left(h x_{2 m(k)}, h x_{2 n(k)+1}\right)\right), \phi\left(d_{2 m(k)}\right), \phi\left(d_{2 n(k)+1}\right), \phi\left(\frac { 1 } { 2 } \left[d\left(h x_{2 m(k)}, h x_{2 n(k)+1}\right)\right.\right.\right. \\
& \left.\left.\left.+d\left(f x_{2 m(k)}, g x_{2 n(k)+1}\right)\right]\right)\right\}-w\left(\operatorname { m a x } \left\{\phi\left(d\left(h x_{2 m(k)}, h x_{2 n(k)+1}\right)\right), \phi\left(d_{2 m(k)}\right)\right.\right. \\
& \left.\left.\phi\left(d_{2 n(k)+1}\right), \phi\left(\frac{1}{2}\left[d\left(h x_{2 m(k)}, h x_{2 n(k)+1}\right)+d\left(f x_{2 m(k)}, g x_{2 n(k)+1}\right)\right]\right)\right\}\right)
\end{aligned}
$$

Taking $k \rightarrow \infty$, we deduce that

$$
\begin{gathered}
\psi(\epsilon) \leq \max \left\{\phi(\epsilon), \phi(0), \phi(0), \phi\left(\frac{1}{2}[\epsilon+\epsilon]\right)\right\}-w\left(\max \left\{\phi(\epsilon), \phi(0), \phi(0), \phi\left(\frac{1}{2}[\epsilon+\epsilon]\right)\right\}\right) \\
\psi(\epsilon) \leq \phi(\epsilon)-w(\phi(\epsilon))<\phi(\epsilon)
\end{gathered}
$$

a contradiction and hence $\left\{h x_{n}\right\}$ is Cauchy sequence. Since $X$ is asymptotically $h$-complete implies that the sequence $\left\{h x_{n}\right\}$ converges to a point $z \in X$.We infer that $\left\{h x_{2 n}\right\}$ and $\left\{h x_{2 n+1}\right\}$ also converges to $z$. $h$ is asymptotically continuous implies that

$$
h h x_{2 n} \rightarrow h z, h h x_{2 n+1} \rightarrow h z, h f x_{2 n} \rightarrow h z, h g x_{2 n+1} \rightarrow h z \text { as } n \rightarrow \infty .
$$

Now using weakly commutativity and sub additivity of $\psi$, we have

$$
\begin{aligned}
\psi\left(d\left(f h x_{2 n}, h z\right)\right) & \leq \psi\left(d\left(f h x_{2 n}, h f x_{2 n}\right)\right)+\psi\left(d\left(h f x_{2 n}, h z\right)\right) \\
& \leq \psi\left(d\left(f x_{2 n}, h x_{2 n}\right)\right)+\psi\left(d\left(h f x_{2 n}, h z\right)\right)
\end{aligned}
$$

Taking $n \rightarrow \infty$ implies that $f h x_{2 n} \rightarrow h z$. Similarly $g h x_{2 n+1} \rightarrow h z$. Suppose $d(h z, g z)>0$. Then

$$
\begin{aligned}
\psi(d(h z, g z)) \leq & \psi\left(d\left(h z, f h x_{2 n}\right)\right)+\psi\left(d\left(f h x_{2 n}, g z\right)\right) \\
\leq & \psi\left(d\left(h z, f h x_{2 n}\right)\right)+\max \left\{\phi\left(d\left(h h x_{2 n}, h z\right)\right), \phi\left(d\left(h h x_{2 n}, f h x_{2 n}\right)\right), \phi(d(h z, g z)),\right. \\
& \left.\phi\left(\frac{1}{2}\left[d\left(h h x_{2 n}, h z\right)+d\left(f h_{2 n}, g z\right)\right]\right)\right\}-w\left(\operatorname { m a x } \left\{\phi\left(d\left(h h x_{2 n}, h z\right)\right), \phi\left(d\left(h h x_{2 n}, f h x_{2 n}\right)\right),\right.\right. \\
& \left.\left.\phi(d(h z, g z)), \phi\left(\frac{1}{2}\left[d\left(h h x_{2 n}, h z\right)+d\left(f h_{2 n}, g z\right)\right]\right)\right\}\right)
\end{aligned}
$$

Taking $n \rightarrow \infty$, we infer that

$$
\psi(d(h z, g z)) \leq \phi(d(h z, g z))
$$

a contradiction.Hence $h z=g z$. Similarly we can easily prove that $h z=f z$.Next we have to show that $z$ is fixed point of $h$. Suppose that $d(h z, z)>0$.From (5), we have

$\psi\left(d\left(f x_{2 n}, g h x_{2 n}\right)\right) \leq \max \left\{\phi\left(d\left(h x_{2 n}, h h x_{2 n}\right)\right), \phi\left(d\left(h x_{2 n}, f x_{2 n}\right)\right), \phi\left(d\left(h h x_{2 n}, g h x_{2 n}\right)\right)\right.$, 


$$
\begin{aligned}
& \left.\phi\left(\frac{1}{2}\left[d\left(h x_{2 n}, h h x_{2 n}\right)+d\left(f x_{2 n}, g h x_{2 n}\right)\right]\right)\right\}-w\left(\operatorname { m a x } \left\{\phi\left(d\left(h x_{2 n}, h h x_{2 n}\right)\right), \phi\left(d\left(h x_{2 n}, f x_{2 n}\right)\right)\right.\right. \\
& \left.\left.\phi\left(d\left(h h x_{2 n}, g h x_{2 n}\right)\right), \phi\left(\frac{1}{2}\left[d\left(h x_{2 n}, h h x_{2 n}\right)+d\left(f x_{2 n}, g h x_{2 n}\right)\right]\right)\right\}\right)
\end{aligned}
$$

Taking $n \rightarrow \infty$, we infer that

$$
\begin{aligned}
\psi(d(z, h z)) \leq & \max \left\{\phi(d(z, h z)), \phi(0), \phi(0), \phi\left(\frac{1}{2}[d(z, h z)+d(z, h z)]\right)\right\} \\
& -w\left(\max \left\{\phi(d(z, h z)), \phi(0), \phi(0), \phi\left(\frac{1}{2}[d(z, h z)+d(z, h z)]\right)\right\}\right)
\end{aligned}
$$

implies that

$$
\psi(d(z, h z)) \leq \phi(d(z, h z))-w(\phi(d(z, h z)))<\phi(d(z, h z))
$$

a contradiction.Hence $h z=z$. Therefore $f z=g z=h z=z$. i.e. $z$ is common fixed point of $f, g$ and $h$.

Finally, we show that $z$ is unique fixed point of $f, g \& h$. Suppose $z^{\prime}$ be another fixed point. Then from (5), we obtain

$$
\begin{aligned}
\psi\left(d\left(f z, g z^{\prime}\right)\right) \leq & \max \left\{\phi\left(d\left(h z, h z^{\prime}\right)\right), \phi(d(h z, f z)), \phi\left(d\left(h z^{\prime}, g z^{\prime}\right)\right), \phi\left(\frac{1}{2}\left[d\left(h z, h z^{\prime}\right)+d\left(f z, g z^{\prime}\right)\right]\right)\right\} \\
& -w\left(\max \left\{\phi\left(d\left(h z, h z^{\prime}\right)\right), \phi(d(h z, f z)), \phi\left(d\left(h z^{\prime}, g z^{\prime}\right)\right), \phi\left(\frac{1}{2}\left[d\left(h z, h z^{\prime}\right)+d\left(f z, g z^{\prime}\right)\right]\right)\right\}\right)
\end{aligned}
$$

we infer that

$$
\psi\left(d\left(h z, h z^{\prime}\right)\right) \leq \phi\left(d\left(h z, h z^{\prime}\right)\right)-w\left(\phi\left(d\left(h z, h z^{\prime}\right)\right)\right)<\phi\left(d\left(h z, h z^{\prime}\right)\right)
$$

a contradiction. Hence $z=z^{\prime}$.

Corollary 2. Let $(X, d)$ be a complete metric space and $f, g$ and $h$ be self mappings on $X$ such that $f(X) \cup g(X) \subseteq h(X)$.If there exists a $w \in W$ satisfying (5).Then the pair $(f, h)$ and $(g, h)$ have a coincidence point in $X$, provided that (i) $h$ is continuous and (iii) $h$ commutes with both $f$ and $g$.Further $f, g$ and $h$ have a unique common fixed point in $X$.

Taking $\psi(t)=t=\phi(t)$,in cor.2 we obtain the following 
Corollary 3. Let $(X, d)$ be a complete metric space. Let $f, g$ and $h$ be self maps on $X$ such that $f(X) \cup g(X) \subseteq h(X)$ and $h$ is continuous and commute with both $f$ and $g$. If there exists a $w \in W$ satisfying the following condition:

$$
\begin{aligned}
d(f x, g y) & \leq \max \left\{d(h x, h y), d(h x, f x), d(h y, g y), \frac{1}{2}[d(h x, h y)+d(f x, g y)]\right\} \\
& -w\left(\max \left\{d(h x, h y), d(h x, f x), d(h y, g y), \frac{1}{2}[d(h x, h y)+d(f x, g y)]\right\}\right)
\end{aligned}
$$

for all $x, y \in x$. Then the pair $(f, h)$ and $(g, h)$ have coincidence point, further $f, g$ and $h$ have unique common fixed point in $X$.

Taking $h=I$, in cor.3 we gain the following

Corollary 4. Let $(X, d)$ be a complete metric space. Let $f$ and $g$ be self maps on $X$. If there exists a $w \in W$ satisfying the following condition:

$$
\begin{aligned}
d(f x, g y) & \leq \max \left\{d(x, y), d(x, f x), d(y, g y), \frac{1}{2}[d(x, y)+d(f x, g y)]\right\} \\
& -w\left(\max \left\{d(x, y), d(x, f x), d(y, g y), \frac{1}{2}[d(x, y)+d(f x, g y)]\right\}\right)
\end{aligned}
$$

for all $x, y \in x$. Then $f$ and $g$ have unique common fixed point in $X$.

\section{An Application}

Throughout in this section,let $X$ and $Y$ be Banach spaces $S \subseteq X$ be the state space and $D \subseteq Y$ be decision space. $\mathrm{B}(\mathrm{S})$ denotes the set of all real-valued bounded functions on $S$.Put $d(a, b)=\sup _{x \in S}|a(x)-b(x)|, \forall a, b \in B(S)$.It is obvious that $(B(S), d)$ is a complete metric space. Define $u: S \times D \rightarrow R, T: S \times D \rightarrow S$ and $H_{i}: S \times D \times R \rightarrow R$ for $i=\{1,2,3\}$.

Now we study those conditions which guarantee the existence and uniqueness of common solutions of functional equations (7).

Theorem 3. If the following conditions are satisfied $\left(C_{1}\right) u$ and $H_{i}$ are bounded for $i=\{1,2,3\}$

$\left(C_{2}\right)$ there exist $\phi \in \Phi, \psi \in \Psi$ and $w \in W$ satisfying

$$
\begin{aligned}
\psi \mid H_{1}(x, y, a(t)) & -H_{2}(x, y, b(t)) \mid \\
& \leq \max \left\{\phi(d(h a, h b)), \phi(d(h a, f a)), \phi(d(h b, g b)), \phi\left(\frac{1}{2}[d(h a, h b)+d(f a, g b)]\right)\right\} \\
& -w\left(\max \left\{\phi(d(h a, h b)), \phi(d(h a, f a)), \phi(d(h b, g b)), \phi\left(\frac{1}{2}[d(h a, h b)+d(f a, g b)]\right)\right\}\right)
\end{aligned}
$$


for all $(x, y) \in S \times D ; a, b \in B(S)$ and $t \in S$. Where $f, g$ and $h$ are defined as follows: for all $x \in S, a_{i} \in B(S)$ and $i=\{1,2,3\}$

$$
\begin{aligned}
& f\left(a_{1}(x)\right)=\operatorname{opt}_{y \in D}\left\{u(x, y)+H_{1}\left(x, y, a_{1}(T(x, y))\right)\right\} \\
& g\left(a_{2}(x)\right)=\operatorname{opt}_{y \in D}\left\{u(x, y)+H_{2}\left(x, y, a_{2}(T(x, y))\right)\right\} \\
& h\left(a_{3}(x)\right)=\operatorname{opt}_{y \in D}\left\{u(x, y)+H_{3}\left(x, y, a_{3}(T(x, y))\right)\right\}
\end{aligned}
$$

$\left(C_{3}\right) f(B(S)) \cup g(B(S)) \subseteq h(B(S))$ and $h$ is asymptotically continuous and weakly commute with both $f$ and $g$.

Then the system of functional equations possess a unique common solution in $B(S)$.

Proof From $\left(C_{1}\right)$ and $\left(C_{2}\right), f, g$ and $h$ be self maps on $B(S)$. Let $a, b \in B(S)$ and $x \in S$.For any $\epsilon>0$ there exist $y, z \in D$ satisfying

$$
\begin{aligned}
& f(a(x))<u(x, y)+H_{1}(x, y, a(T(x, y)))+\epsilon \\
& g(b(x))<u(x, z)+H_{2}(x, z, b(T(x, z)))+\epsilon \\
& f(a(x)) \geq u(x, z)+H_{1}(x, z, a(T(x, z)))+\epsilon \\
& g(b(x)) \geq u(x, y)+H_{2}(x, y, b(T(x, y)))+\epsilon
\end{aligned}
$$

Combining above inequalities with $\left(C_{2}\right)$, we obtain the following:

$$
\begin{aligned}
\psi(|f(a(x))-g(b(x))|) \leq & \psi(\epsilon)+\psi\left(\operatorname { m a x } \left\{\left|H_{1}(x, y, a(T(x, y)))-H_{2}(x, y, b(T(x, y)))\right|,\right.\right. \\
& \left.\left.\left|H_{1}(x, z, a(T(x, z)))-H_{2}(x, z, b(T(x, z)))\right|\right\}\right) \\
\leq & \psi(\epsilon)+\max \left\{\phi(d(h a, h b)), \phi(d(h a, f a)), \phi(d(h b, g b)), \phi\left(\frac{1}{2}[d(h a, h b)+d(f a, g b)]\right)\right\} \\
- & w\left(\max \left\{\phi(d(h a, h b)), \phi(d(h a, f a)), \phi(d(h b, g b)), \phi\left(\frac{1}{2}[d(h a, h b)+d(f a, g b)]\right)\right\}\right)
\end{aligned}
$$

Letting $\epsilon \rightarrow \infty$ we get

$$
\begin{aligned}
\psi(|f(a(x))-g(b(x))|) & \leq \max \left\{\phi(d(h a, h b)), \phi(d(h a, f a)), \phi(d(h b, g b)), \phi\left(\frac{1}{2}\right)[d(h a, h b)+d(f a, g b)]\right\} \\
& -w\left(\max \left\{\phi(d(h a, h b)), \phi(d(h a, f a)), \phi(d(h b, g b)), \phi\left(\frac{1}{2}[d(h a, h b)+d(f a, g b)]\right)\right\}\right)
\end{aligned}
$$

Therefore, Theorem 3.1 ensures that $f, g$ and $h$ have a unique common fixed point in $\mathrm{B}(\mathrm{S})$. That is, the system of functional equations (7) possesses a unique common solution $\mathrm{B}(\mathrm{S})$. Similarly we can change the condition $\left(C_{2}\right)$ in Theorem 3.1 and get the common solution using corollaries. Taking $h=I$ in Theorem, we conclude that 
Theorem 4. Let the following condition hold: $\left(C_{4}\right) u$ and $H_{i}$ are bounded for $i \in\{1,2\}$. $\left(C_{5}\right)$ there exist a $w \in\{W\}$ satisfying

$$
\begin{aligned}
\left|H_{1}(x, y, a(t))-H_{2}(x, y, b(t))\right| \leq & \left.\max \left\{d(a, b), d(a, f a), d(b, g b), \frac{1}{2}[d(a, b)+d(f a, g b)]\right)\right\} \\
& \left.-w\left(\max \left\{d(a, b), d(a, f a), d(b, g b), \frac{1}{2}[d(a, b)+d(f a, g b)]\right)\right\}\right)
\end{aligned}
$$

for all $(x, y) \in S \times D ; a, b \in B(S)$ and $t \in S$. Where $f$ and $g$ are defined as follows: for all $x \in S, a_{i} \in B(S)$ and $i=\{1,2,3\}$

$$
\begin{aligned}
& f\left(a_{1}(x)\right)=\operatorname{opt}_{y \in D}\left\{u(x, y)+H_{1}\left(x, y, a_{1}(T(x, y))\right)\right\} \\
& g\left(a_{2}(x)\right)=\operatorname{opt}_{y \in D}\left\{u(x, y)+H_{2}\left(x, y, a_{2}(T(x, y))\right)\right\}
\end{aligned}
$$

for all $x \in S, a_{1}, a_{2} \in B(S)$. Then the system of functional equations

$$
\begin{aligned}
& f(x)=o p t_{y \in D}\left\{u(x, y)+H_{1}(x, y, f(T(x, y)))\right\} \\
& g(x)=o_{y \in D}\left\{u(x, y)+H_{2}(x, y, g(T(x, y)))\right\}
\end{aligned}
$$

possesses a unique common solution in $B(S)$.

\section{Acknowledgements}

The first author is thankful to the National Board for Higher Mathematics(NBHM),India for providing financial assistance during this research study.

\section{References}

[1] R. Baskaran and P. V. Subrahmanyam. A note on the solution of a class of functional equations principle. Appl. Anal,volume 22 (3-4); pages 235-241, 1986.

[2] R. Bellman. Dynamic Programming, Princeton University Press. Princeton, New Jersey, 1957.

[3] R. Bellman and E. S. Lee. Functional equations in dynamic programming. Aequationes Math. 17(1):1-18, 1978.

[4] P. C. Bhakta and S. R. Choudhury. Some existence theorems for functional equations arising in dynamic programming. II J. Math. Anal. Appl.,131(1);217-231, 1988.

[5] P. C. Bhakta and S. Mitra. Some existence theorems for functional equations arising in dynamic programming J. Math. Anal. Appl.,98(2):348-362, 1984. 
[6] F.E Brawder, W.V. Petryshyn. Contraction of fixed points of nonlinear mappings in Hilbert space J. Math. Anal. Appl, 20:197-228,1967.

[7] S. S. Chang and Y. H. Ma. Coupled fixed points for mixed monotone condensing operators and an existence theorem of the solutions for a class of functional equations arising in dynamic programming J. Math. Anal. Appl, 160(2):468-479,1991.

[8] P.Chumki and A.P. Baisnab. Asymptotically regularity and fixed point theorems The math. student, 46:54-59,1978.

[9] Lj.B. Ciric. A generalization of Banachs contraction principle Proc. Am. Math. Soc., 45:267-273, 1974.

[10] B. Fisher. Results on common fixed points on bounded metric spaces Math. sem. Notes, 7:73-80, 1979.

[11] M. D. Guay, K. L. Singh. Fixed points of asymptotically regular mappings Math. vesnik,35:101-106, 1983.

[12] K.P.R. Sastry, S.R. Naidu, I.H.N. Rao and K.P.R. Rao. Common fixed point for asymptotically regular mappings Int.J.Pure Appl. Math.,15:849-854, 1984.

[13] S.L.Singh and S.P. Singh. A fixed point theorems Indian J. pure appl. Math.,11:15841586, 1980.

[14] P.L. Sharma and A.K. Yud. Fixed point theorems under asymptotic regularity at a point II Jnanabha,11:127-131, 1981.

[15] Z. Liu. A note on unique common fixed point Bull. Calcutta Math. Soc.,85(5):469$472,1993$.

[16] Z. Liu. Existence theorems of solutions for certain classes of functional equations arising in dynamic programming, J. Math. Anal. Appl. 262 (2): 529-553, 2001.

[17] Z. Liu. Coincidence theorems for expansion mappings with applications to the solutions of functional equations arising in dynamic programming, Acta Sci. Math. (Szeged) 65 (1): 359-369,1999.

[18] Z. Liu. Compatible mappings and fixed points, Acta Sci. Math. (Szeged) 65(2), 371-383,1999.

[19] Z. Liu, R. P. Agarwal, and S. M. Kang On solvability of functional equations and system of functional equations arising in dynamic programming, J. Math. Anal. Appl. 297 (1), 111-130,2004.

[20] Z. Liu and J. S. Ume On properties of solutions for a class of functional equations arising in dynamic programming, J. Optim. Theory Appl. 117 (3), 533-551,2003. 
[21] H. K. Pathak and B. Fisher Common fixed point theorems with applications in dynamic programming, Glas. Mat. Ser. III 31(51)(2), 321-328,1996.

[22] B. N. Ray On common fixed points in metric spaces, Indian J. Pure Appl. Math. 19 (10), 960-962,1988.

[23] B. E. Rhoades, S. Sessa, M. S. Khan and M. Swaleh On fixed point of asymptotically regular mappings, J. Austral. Math. Soc. (Series A) 43, 328-346,1987.

[24] S. S. Zhang Some existence theorems of common and coincidence solutions for a class of systems of functional equations arising in dynamic programming, Appl. Math. Mech. 12 (1), 31-37,1991. 